\title{
Application of Neuro Fuzzy for Automatic Switching of Steam Turbine in a CCPP
}

\author{
Arnika soni, ${ }^{1}$ Prof. Hemant Amhia ${ }^{2}$ \\ M.E. Control system Jabalpur Engineering college, Jabalpur, (M.P) \\ Department of Electrical Jabalpur Engineering College, Jabalpur, (M.P)
}

\begin{abstract}
In electricity generation power plants lot of heat is generated when a GAS turbine is applied to generate electricity, this excessive heat is wasted and to avoid the wastage of heat steam turbines are added in sequence to heat the boilers, which generates steam. This steam is further utilized to generate electricity; this operation is named as co-generation power plant. Such a system when operated manually has a drawback that steam turbine cannot operate regularly due to varying heat generation by the GAS turbine. An automation system must be applied to operate steam turbine so that undue manual delays in switching on/off of steam turbine can be avoided. In this paper I am proposing a Neuro Fuzzy based system which will operate the steam turbine on the basis of the historical data.

Neuro Fuzzy approach is becoming more and more popular in research as it uses the background knowledge to determine the future states of the system. To best utilize the energy and reduce the losses, an automation system can be applied to trip the steam turbine on/off which will be fully utilizing the waste energy. There can be several ways such automation can be applied. In this paper I am proposing such an automation system which will be best on the total efficiency of the turbines calculated and supplied through a Neuro Fuzzy controller.

The heat rejected by the higher temperature cycle is recovered and used by a lower temperature cycle to produce additional power to realize an improved overall efficiency. For the combination, the separate cycles must operate on separate fluids. I am proposing a Neuro-Fuzzy based Hybrid Modeling System which will produce the outputs on the basis of the historical data of the plant finding when to switch on/off the secondary turbine. The outputs can be attached to an automatic system so that manual operations of the turbine control can also be eliminated. Inclusion of Neuro-Fuzzy not only makes the accurate and quick decision but also uses the fresh data of the plant to make the decisions related with switch operations.

The heat rejected by Gas turbine is highly appreciable as the exhaust temperature varies from $4500 \mathrm{C}$ to $6500 \mathrm{C}$ depending upon pressure ratio and turbine inlet temperature. This energy goes as waste if it is rejected to atmosphere. This waste heat energy may be utilized to produce steam in a heat recovery steam generator (HRSG) and may be expanded in steam turbine to develop additional power based on Rankine cycle. This combination is termed as Gas/Steam combined cycle power plant. The Gas cycle and steam cycle may use separate generator or a single generator. It is desirable that the exhaust temperature of Gas turbine should be above $5700 \mathrm{C}$ otherwise the steam cycle will be inefficient resulting in lower combined cycle plant efficiency.
\end{abstract}

Keywords:- Neuro Fuzzy, Automation, Combined Cycle Power Plant, Hybrid system.

\section{INTRODUCTION}

Gas/Steam combined cycle efficiency ranges from $47 \%$ to $60 \%$ as compared to $35 \%$ to $40 \%$ of conventional steam or gas turbine power plant.

Having CCPP is undoubtedly increases the efficiency and uses the waste heat to a great extent. They also have the requirement to switch on or off the secondary steam power plant as per the available heat in the primary power plant. In this paper, an automation system is being proposed to efficient operations of CCPPs using Neuro-Fuzzy System and Hybrid Approach. Hybrid System has been applied for achieving systems evolution according to continuous dynamics, discrete dynamics, and logic rules. The possibility of turning on/off the gas and steam turbine, the operating constraints (minimum up and down times) and the different types of start up of the turbines characterize the hybrid behavior of a combined cycle power plant [1].

The electric power industry has been subject to deep changes in structure and organization. From the technological side, the use of combined cycle power plants (CCPP) became more and more popular because of their high efficiency. A typical CCPP is composed of a gas cycle and a steam cycle. The gas cycle is fed by fuel and produces electric power through the expansion of the gas in the gas turbine; the steam cycle is supplied with the output exhaust gas from the gas turbine and generates both electricity and steam for the industrial processes. From the economic side, the liberalization of the energy market promoted the need of operating CCPPs in the most efficient way, which is by maximizing the profits due to the sales of steam and electricity and by minimizing the operating costs.

A major problem of optimizing the short-term operation of a CCPP, i.e. to optimize the plant on an hourly basis over a time horizon that may vary from few hours to one day as proposed by the authors. A large stream of research in the power systems area focused on this problem. The usual recipe is to recast the economic optimization into the minimization of a cost functional and to account for the physical model of the plant through suitably defined constraints. The results 
available in the literature differ both in the features of the CCPP modeled and in the scope of optimization.

CCPPs are efficient and more power generators than the single power plants. Application of CCPPs has become wide spread and due to their advantages they are being accepted and implemented. Along with several advantages CCPPs offer, they have certain troubles in operating them efficiently. The most critical of these is operating the secondary Turbine on/off as per the heat generation by the primary turbine.

The aim of my research is to show how both the tasks of modeling and optimization of CCPPs can be efficiently solved by resorting to hybrid system methodologies. The research focused on the investigation of basic properties such as stability, controllability and observability. For achieving the goal, I have added a Neuro-Fuzzy system along with the hybrid system, so that the better stability and control can be evaluated automatically on the basis of the historical data.

I use discrete-time hybrid systems in the Mixed Logical Dynamical (MLD) form for two reasons. First, they provide a general framework for modeling many discrete features of CCPPs, including the coordination and prioritization between different devices; second, they are suitable to be used in online optimization schemes.

\section{REVIEW OF THE EXISTING SYSTEM}

\section{Neuro Fuzzy Controls and Their Applications}

Neuro Fuzzy approach applied in modeling the co-generation power plant with hybrid system in MLD form will produce a proper modeling of the CGPP for optimization and for prioritization of the engines for best outputs.

Neuro Fuzzy modeling is concerned with the extraction of models from numerical data representing the behavior of a system. The models in this case are rule-based and use the formalism of fuzzy logic, i.e. they consists of sets of fuzzy "if - then" rules with possibly several premises.

The fuzzy logic controller is very attractive for ill-defined systems or systems with uncertain parameters. With the help of fuzzy logic concepts, expert knowledge can be used directly to design a controller. Fuzzy logic allows one to express the knowledge with subjective concepts such as very large, too small, which are mapped to numeric ranges [11].

Similarities exist between the NNs and the FCs. Both techniques allow interpolate reasoning which makes them free from the true/false restriction of conventional logic systems [12]. In a multi-layer neural network of feed-forward type, input nodes record the features and pass activation values to the output layer through a hidden layer. The addition of the hidden layers to the two-layer perception networks allows these networks to represent any continuous mapping from input to output [13]. An appropriate training technique, adjusts the connection weights of the network to improve the match between the output of the network and the correct results. To design the FC some variables which can represent the dynamic performance of the system should be chosen to be fed as the inputs.
The basic idea of combining fuzzy systems and neural networks is to design an architecture that uses a fuzzy system to represent knowledge in an interpretable manner and the learning ability of a neural network to optimize its parameters. The drawbacks of both of the individual approaches - the black box behavior of neural networks, and the problems of finding suitable membership values for fuzzy systems - could thus be avoided. A combination can constitute an interpretable model that is capable of learning and can use problem-specific prior knowledge. Therefore, Neuro-fuzzy methods are especially suited for applications, where user interaction in model design or interpretation is desired.

A Neuro-fuzzy system can be viewed as a 3-layer feed forward neural network. The first layer represents input variables, the middle (hidden) layer represents fuzzy rules and the third layer represents output variables. Fuzzy sets are encoded as (fuzzy) connection weights. It is not necessary to represent a fuzzy system like this to apply a learning algorithm to it. However, it can be convenient, because it represents the data flow of input processing and learning within the model.

The optimization of a combined cycle power plant is accomplished by exploiting hybrid systems, i.e. systems evolving according to continuous dynamics, discrete dynamics, and logic rules. The possibility of turning on/off the gas and steam turbine, the operating constraints (minimum up and down times) and the different types of start up of the turbines characterize the hybrid behavior of a combined cycle power plant. In order to model both the continuous/discrete dynamics and the switching between different operating conditions we use the framework of Mixed Logic Dynamical systems.

Neuro Fuzzy approach applied in modeling the co-generation power plant with hybrid system in MLD form will produce a proper modeling of the CGPP for optimization and for prioritization of the engines for best outputs.

\section{ANFIS Model}

Neuro-fuzzy modeling is concerned with the extraction of models from numerical data representing the behavior of a system. The models in this case are rule-based and use the formalism of fuzzy logic, i.e. they consists of sets of fuzzy "if - then" rules with possibly several premises.

\section{PLANT HYBRID FEATURES}

The features which suggest modeling the Island power plant as a hybrid system are the following:

- the presence of the binary inputs ul1 and ul2;

- the turbines have different start up modes, depending on how long the turbines have been kept off;

- Electric power, steam flow and fuel consumption are continuous valued quantities evolving with time.

Furthermore, the following constraints have to be taken into account: 
- the operating constraints on the minimum amount of time for which the turbines must be kept on/off (the so-called minimum up/down times);

- The priority constraint (2). This condition, together with the previous one, leads to constraints on the sequences of logic inputs which can be applied to the system;

- The gas turbine load u1 and the steam mass flow u2 are bounded.

\section{MLD Form for Hybrid Systems}

The derivation of the MLD form of a hybrid system involves basically three steps. The first one is to associate with a logical statement $\mathrm{S}$, that can be either true or false, a binary variable $\delta \in\{0,1\}$ that is 1 if and only if the statement holds true. Then, the combination of elementary statements S1, Sq into a compound statement via the Boolean operators AND $(\wedge)$, OR (V), NOT $(\sim)$ can be represented as linear inequalities over the corresponding binary variables

$$
\delta i, i=1, \ldots, q \text {. }
$$

An example would be the condition aT $\mathrm{x} \leq 0$ :

$$
\mathrm{aT} \mathrm{x} \leq 0 \Leftrightarrow[\delta=1]
$$

where $\mathrm{x} \in \mathrm{X} \subseteq \mathrm{Rn}$ is a continuous variable and $\mathrm{X}$ is a compact set. If one defines $\mathrm{m}$ and $\mathrm{M}$ as lower and upper bounds on aT $\mathrm{x}$ respectively, the inequalities

$$
\begin{aligned}
& \text { aT } \mathrm{x} \leq \mathrm{M}-\mathrm{M} \delta \\
& \text { aT } \mathrm{x} \geq \varepsilon+(\mathrm{m}-\varepsilon) \delta
\end{aligned}
$$

assign the value $\delta=1$ if and only if the value of $\mathrm{x}$ satisfies the threshold condition.

Note that $\varepsilon>0$ is a small tolerance (usually close to the machine precision) introduced to replace the strict inequalities by non-strict ones.

The second step is to represent the product between linear functions and logic variables by introducing an auxiliary variable $\mathrm{z}=\delta \mathrm{aT} \mathrm{x}$. Equivalently, $\mathrm{z}$ is uniquely specified through the mixed integer linear inequalities

$$
\begin{aligned}
& \mathrm{z} \leq \mathrm{M} \delta \\
& \mathrm{z} \geq \mathrm{m} \delta \\
& \mathrm{z} \leq \mathrm{aT} \mathrm{x}-\mathrm{m}(1-\delta) \\
& \mathrm{z} \geq \mathrm{aT} \mathrm{x}-\mathrm{M}(1-\delta)
\end{aligned}
$$

The third step is to include binary and auxiliary variables in an LTI discrete time dynamic system in order to describe in a unified model the evolution of the continuous and logic components of the system.

\section{APPLICATION OF HYBRID SYSTEM IN CGPP}

The cogeneration combined cycle power plant Island consists of four main components: a gas turbine, a heat recovery steam generator, a steam turbine and a steam supply for a paper mill. Simplified input/output description of the plant presented in Figure. Heat recovery steam generator does not appear in Figure because it is hidden in the "steam turbine" block. The plant has two continuous-valued inputs ( $\mathrm{u} 1$ and $\mathrm{u} 2$ ), and two binary inputs (ul1 and ul2): $-\mathrm{u} 1$ is the set point for the gas turbine load (in percent). The permitted operation range for the gas turbine is in the interval [u1,min, u1, max];

$-\mathrm{u} 2$ is the steam mass flow to the paper mill. The permitted range for the steam flow is in the interval [u2,min, u2,max];

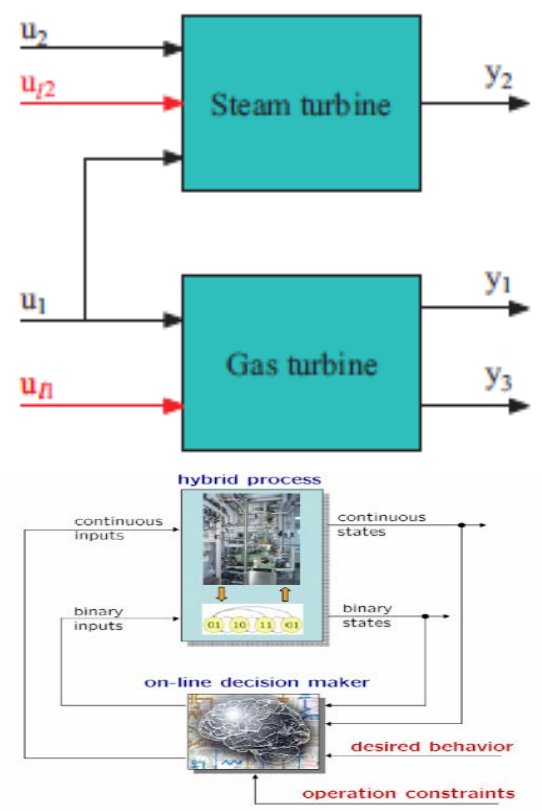

\section{PROPOSED ALGORITHM}

My research is focused on use of Neuro-Fuzzy System applied with Hybrid System in a Combine Cycle Power Plant. I am proposing the following flow of the system to evaluate control and stability of the plant:

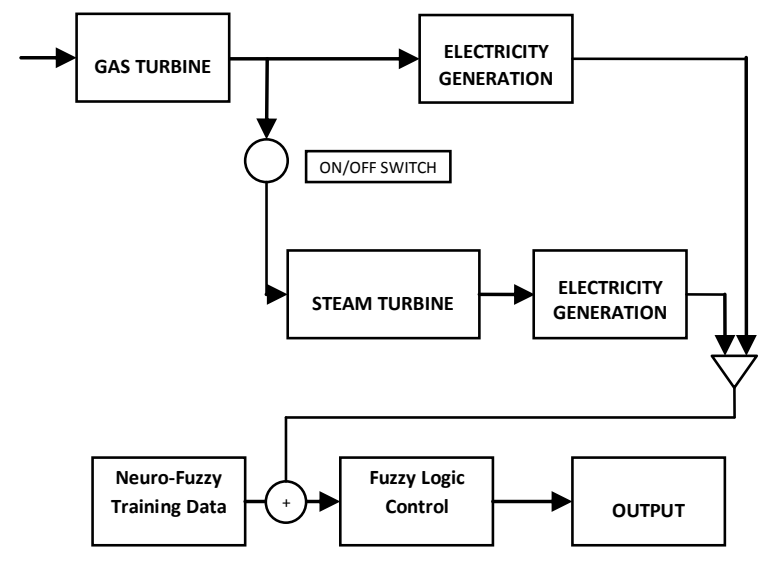

I have collected the data for input of the fuzzy logic control [Table 1]. 
Efficiency of the Gas Turbine is used as input to on/off switch along with output of the fuzzy logic controller which uses the industrial output of the existing system to control on/off switch. If the output of fuzzy is high then the switch remains on but when the output becomes low, switch is set of off which will automatically stop the steam turbine.

The input of the fuzzy logic controller is historical data and current efficiency of the Gas turbine, which are used to decide whether the steam turbine should be operated or not. I have proposed the following rule set for the fuzzy logic controller for the purpose of simulation.

1. When Efficiency of Gas Turbine is low and historical input is low then output is off.

2. When Efficiency of Gas Turbine is moderate and historical input is medium then output is on.

3. When Efficiency of Gas Turbine is High and historical input is high then output is on

Table 1. Data taken from a Running Cogeneration Power Plant at Surat, Gujrat.

\begin{tabular}{|l|l|}
\hline EFFICIENCY & OUTPUT \\
\hline 00.00 & 0 \\
\hline 30.00 & 0 \\
\hline 43.87 & 0 \\
\hline 44.30 & 1 \\
\hline 44.29 & 1 \\
\hline 41.90 & 0 \\
\hline 44.12 & 1 \\
\hline 43.83 & 1 \\
\hline 43.47 & 0 \\
\hline 43.80 & 1 \\
\hline 43.23 & 0 \\
\hline 43.16 & 0 \\
\hline 43.96 & 1 \\
\hline 42.77 & 0 \\
\hline 42.35 & 0 \\
\hline 42.22 & 0 \\
\hline 42.84 & 0 \\
\hline 43.52 & 0 \\
\hline 44.12 & 1 \\
\hline 44.67 & 1 \\
\hline 44.41 & 1 \\
\hline 44.18 & 1 \\
\hline 43.96 & 1 \\
\hline 43.51 & 0 \\
\hline 43.90 & 1 \\
\hline 43.49 & 0 \\
\hline 41.37 & 0 \\
\hline 42.90 & 0 \\
\hline 44.20 & 1 \\
\hline 44.55 & 1 \\
\hline 43.68 & 0 \\
\hline 43.88 & 0 \\
\hline & \\
\hline & \\
\hline
\end{tabular}

\begin{tabular}{|l|l|}
\hline 43.26 & 0 \\
\hline 50.00 & 0 \\
\hline 75.00 & 1 \\
\hline 80.00 & 1 \\
\hline 100.00 & 1 \\
\hline
\end{tabular}

Operation of the Gas Turbine and Steam Turbine are mapped using random generation control of the MATLAB for simulation purposes, which generates the efficiency output of the each turbine between the 0 to 50 for Gas Turbine and 0 to 35 for Steam Turbine. Gas Turbine feeds Heat to Steam Turbine for its operations. An on/off switch is can be applied between Gas and Steam Turbine which can be operated on the basis of the output of Fuzzy Logic Control. Combined efficiency output of the Gas and Steam turbine is fed to the Fuzzy logic controller which will provide the output between 0 and 1 i.e. high and low outputs which can be used to control the on/off switch between Gas and Steam Turbines.

\section{RESULTS AND DISCUSSION}

I have proposed a neuro-fuzzy based mechanism to model the system and displayed through simulation that we can get a good high and low outputs to control the secondary power plant from using any binary circuit applied on the outputs. I have used the historical data to evaluate the outputs which is another important feature of my neuro-fuzzy base $\mathrm{d}$ model.

The graph shown below shows the peak values I have got through simulation, which I am proposing to use to operate the steam turbine. This graph shows that when the combined efficiency of the turbines is high then the heat generated by the thermal plant is enough that the steam turbine continues its operations. But when the combined efficiency decrease then a threshold point then the switch can be operated to switch off the working of operation of steam turbine automatically using a relay based circuit.

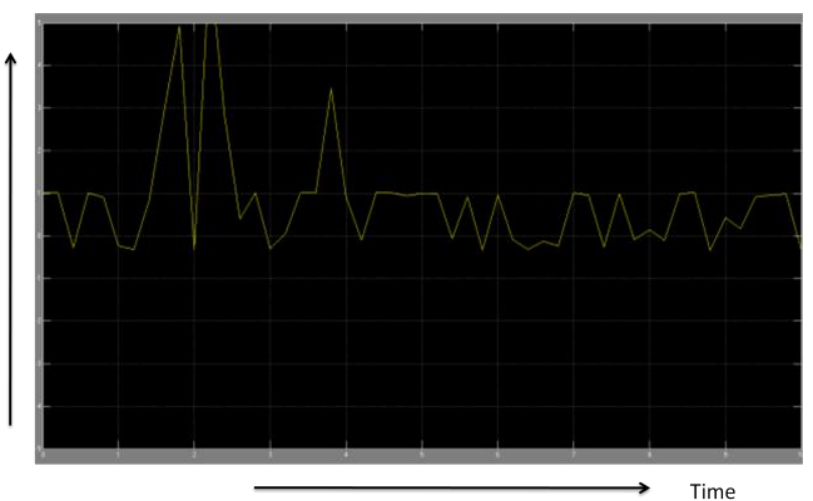

Curve generated from matlab simulation showing the load variation

I have used discrete time hybrid system in the mixed logical dynamical (MLD) form for two reasons :

- First, they provide a general framework for modeling many discrete feature of CCPP's 
- Second, these are suitable to be used in on line optimization schemes

Nero -fuzzy approach applied in modeling of CGPP with hybrid system in MLD from will produce a proper modeling of the CGPP for optimization and for prioritization of the engines for best output.

- In order to illustrate how the startup coefficients are assigned, we focus on the gas turbine, being the procedure analogs for the steam turbine.

- A curve showing the load demand (variations) of consumers with respect to time. The load curve we use is monthly for industrial countries.

- The load curve is usually divided in three parts namely base load, intermediate load and peak load.

- The based load is the load below which the demand falls and is supplied $100 \%$ of the time i.e. off time.

- The peak loss occurs for small period (say 15\% of the day time) on time.

- The remaining load region represents the intermediate load.

- The peak load plant could be gas turbine unit it is always desirable to allow the steam plant to operate as a base load.

From the outputs of the simulation, we control the on/off switch which will provide the automation of the system and hence the manual operations will be avoided. Output graph for the results obtained indicates two states high and low, which is suitable for operating of the system.

I intend to show the task of modeling and optimization of the CCPP i.e. to provide efficient and automatic control of the on/off states of the secondary power plant which works as per the efficiency being produced by the primary power plant.

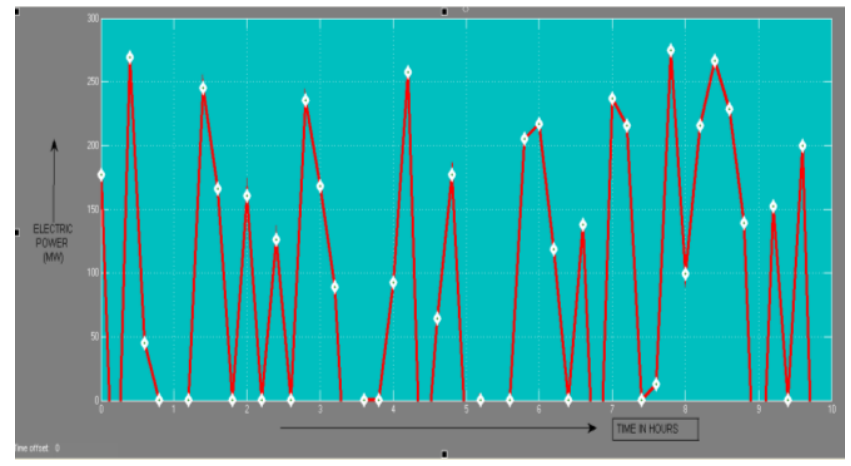

\section{CONCLUSION}

The main goal of this paper is to show that hybrid systems in the MLD form provide a suitable framework for modeling CCPPs. In particular, many features like the possibility of switching on/off the turbines, the presence of minimum up and down times, priority constraints between turbines and different startup procedures can be captured by an MLD model. We point out that also other characteristics, like ramp constraints or nonlinear input/output relations (approximated by piecewise affine functions), can be easily incorporated in the MLD description.

\section{REFERENCES}

[1] Giancarlo Ferrari-Trecate1, Eduardo Gallestey2, Paolo Letizia1, Matteo Spedicato1, Manfred Morari1, and Marc Antoine31 Institut f ur Automatik, ETH - Swiss Federal Institute of Technology, ETL, CH-8092 Z"urich, Switzerland

[2] R. Alur, T. A. Henzinger, and P. H. Ho. Automatic symbolic verification of embedded systems. IEEE Trans. on Software Engineering, 22(3):181-201, March 1996.

[3] J.M. Arroyo and A.J. Conejo. Optimal response of a thermal unit to an electricity spot market. IEEE Trans. on Power Systems, 15(3):1098-1104, 2000.

[4] A. Bemporad, G. Ferrari-Trecate, and M. Morari. Observability and Controllability of Piecewise Affine and Hybrid Systems. IEEE Trans. on Automatic Control, 45(10): 1864-1876, 2000.

[5] A. Bemporad and M. Morari. Control of systems integrating logic, dynamics, and constraints. Automatica, 35(3):407-427, 1999.

[6] M.S. Branicky. Multiple Lyapunov functions and other analysis tools for switched and hybrid systems. IEEE Trans. on Automatic Control, 43(4):475-482, 1998.

[7] M.S. Branicky, W.S. Borkar, and S.K. Mitter. A unified framework for hybrid control: model and optimal control theory. IEEE Trans. on Automatic Control, 43(1):31-45, 1998.

[8] G. Ferrari-Trecate, F.A. Cuzzola, D. Mignone, and M. Morari. Analysis and control with performance of piecewise affine and hybrid systems. Proc. American Control Conference, pages 200-205, 2001.

[9] G. Ferrari-Trecate, E. Gallestey, P. Letizia, M. Spedicato, M. Morari, and M. Antoine. Modeling and control of co-generation power plants: A hybrid system approach. Technical report, AUT01-18, Automatic Control Laboratory, ETH Zurich, 2001.

[10] K. Moslehi, M. Khadem, R. Bernal, and G. Hernandez. Optimization of multiplant cogeneration system operation including electric and steam networks. IEEE Trans. on Power Systems, 6(2):484-490, 1991.

[11] L.A. Zadeh, Outline of a new approach to the analysis of complex systems and decision processes, IEEE Trans. Syst. Man Cybernet. 3 (1) (1973) 28-44.

[12] J.-S.R. Jang, C.-T. Sun, Neuro-Fuzzy modeling and control, IEEE Proc. 83 (3) (1995) 378-406.

[13] L.L. Lai, Intelligent System Applications in Power Engineering: Evolutionary Programming and Neural Networks, Wiley, Chichester, UK, 1998. 